\title{
Climate data analysis to assess resilience of wall assemblies to moisture loads arising from the effects of wind-driven rain
}

\author{
Zhe Xiao ${ }^{1,2, *}$, Michael A. Lacasse ${ }^{1}, A$. Gaur ${ }^{l}$, and Elena Dragomirescu ${ }^{2}$ \\ ${ }^{1}$ National Research Council Canada, Construction Research Centre, 1200 Montreal Road, Ottawa, Ontario, Canada \\ ${ }^{2}$ University of Ottawa, Civil Engineering Department, 75 Laurier Ave E, Ottawa, Ontario, Canada
}

\begin{abstract}
In North America, and abroad, there currently exist standard test protocols for assessing the watertightness of wall assemblies and fenestration components although most of these methods are not directly related to expectations of in-field conditions as might be experienced by a wall assembly over its intended service life. How useful might such test protocols be to help determine the longevity of wall assemblies to future climate loads? Existing walls may, depending on their geographic location, be vulnerable to future climate loads and thus risk premature deterioration. For the design of new wall assemblies consideration ought to given to the non-stationarity of the climate and implications on the moisture loads on walls and the expected performance over the long-term. To permit assessing the resilience of wall assemblies to the effects of a changing climate as may occur in the future, and indeed, perhaps heightened moisture loads, one requires sufficient information on the watertightness of the assembly in relation to specified wind-driven rain loads and wall air-leakage conditions from which wall moisture retention functions could readily be developed. Such moisture functions are the basis of input of moisture loads to hygrothermal models and from which the expected long-term wall moisture performance can subsequently be derived. In this paper, a description is provided of the strategies used to analyze the WDR load for generating experimental input for a watertightness test protocol under development to assess resilience of wall assemblies to moisture loads arising from the effects of wind-driven rain in consideration of both historical climate loads and those as may arise from a changing climate.
\end{abstract}

\section{Introduction}

The building façade plays an important role in maintaining the function and integrity of a building given that it impedes most of the environmental effects acting on the exterior building surface, such as rain, wind, light and noise, as might enter the building envelop. Wall assemblies, as major components of building façades, are constantly subjected to these effects during their lifecycles. Wind-driven rain (WDR), the primary source of the moisture load that could bring detrimental effects to building façade components, is the co-occurrence of rain, in the form of raindrops, and wind. Raindrops of a WDR event have both vertical and horizontal velocity components during the rain event and generally follow the wind flow pattern in the open field. In theory, all rain events can be considered as WDR given the constant movement of air in the atmosphere. The WDR load is identified as the amount of water that is deposited on the vertical surface of the building facade and can be quantified by using the semi-empirical equations [1-3] by knowing the rainfall intensity, wind direction and speed, surrounding conditions, or by taking in-field measurements $[4,5]$.

For that portion of rainwater that reaches the exterior surface of the building façade, it could enter the wall assembly through different deficiencies that in-service are present on and an inherent part of wall assembly. Deficiencies occur either by inappropriate installation of components, or from the process of aging of component materials. The moisture load is derived from water that has infiltrated through deficiencies; the load is affected by multiple factors such as the type of deficiencies, WDR load, wall cavity depths, and air-leakage of wall assemblies. In practice, the moisture load can be evaluated by performing watertightness tests on a wall assembly, for which the WDR load, wind pressure, and properties of test specimen can be controlled and adjusted for simulating different climate conditions. Thereafter, the results from such tests can be used as an initial condition for assessing the long-term performance of the wall assembly.

\subsection{Motivation and objective}

Extreme loads generated from the climate may affect any type of building on any given day of the year. Properly estimating the performance and resilience of wall assemblies subjected to such loads is very important for the design of adequate and long-term moisture performance of buildings constructed in the future and to determine the resilience of wall assemblies to moisture loads of existing buildings. To accurately evaluate the

\footnotetext{
* Corresponding author: jimmyxiao91@gmail.com
} 
future moisture performance of wall assemblies, the National Research Council Canada (NRC) is currently developing criteria for the design of climate resilient building envelops and establishing appropriate test procedures to assess different building envelop configurations. As such, the entire assessment will be carried out through several iterative steps that include: (i) estimate WDR loads on wall assemblies based on climate data; (ii) measure the level of water ingress in and water retention of wall assemblies exposed to selected WDR loads from watertightness tests; (iii) correlate the degree of water retention with the moisture response of wall assemblies; (iv) adjust the strategies to select WDR loads.

In respect to the WDR load, in ISO 15927-3 [3] equations are provided to quantify these loads:

$$
\begin{gathered}
I_{A}=\frac{2}{9} \times \sum v \times R_{h}^{8 / 9} \times \cos (D-\theta) \\
I_{A W}=I_{A} \times C_{R} \times C_{T} \times O \times W
\end{gathered}
$$

Where $\mathrm{v}$ is the hourly mean wind speed at the given height, $\mathrm{R}_{\mathrm{h}}$ is the hourly rainfall intensity, $\mathrm{D}$ is the angle between hourly wind speed directions to the north, and $\theta$ is the wall orientation in regard to the north direction.

To assess the WDR on the building façade, a few more factors must be considered based on the airfield WDR load these include: terrain roughness coefficient $C_{R}$, topography coefficient $\mathrm{C}_{\mathrm{T}}$, obstruction factor $\mathrm{O}$ and wall factor W.

These factors are individually determined for each case investigated. The hourly wind speed, wind direction and hourly rainfall intensity needed to estimate WDR load are obtained from the climate data collected from meteorological stations.

Once the WDR load is determined, watertightness tests, an equivalent WDR load can then be applied to the test specimens using appropriate test facilities, as described hereafter. The new Dynamic Wall Testing Facility (DWTF), as shown in Fig.1, designed and built at the NRC, will be used to fulfill such a task. The DWTF is capable of testing specimens up to $2.44 \mathrm{~m} \times 2.44 \mathrm{~m}$. A large static pressure (e.g. $2.5 \mathrm{kPa}$ ) and an equivalent dynamic pressure can be produced by an industrial blower. Simulated WDR events can be generated and applied to specimens through the use of a water spray system that imitates rain water deposition to the exterior wall surface, and a flap control system integrated to the DWTF that is intended to replicate severe wind action. Water collected from designated locations on the test specimens during the watertightness test will be considered as the moisture load. This moisture load will be used as an input to hygrothermal simulations to permit assessing the longterm performance of wall assemblies.

In this article, methodologies and methods to estimate the WDR loads from climate data in the Ottawa region of Canada will be introduced and discussed. The same strategies may be applied to other locations across Canada. The Ultimate goal of this research is to provide experimental input for watertightness tests. Thereafter, the moisture load derived from the watertightness tests is applied to the simulation to permit evaluating the resilience of the wall assemblies when subjected to the selected climate data.

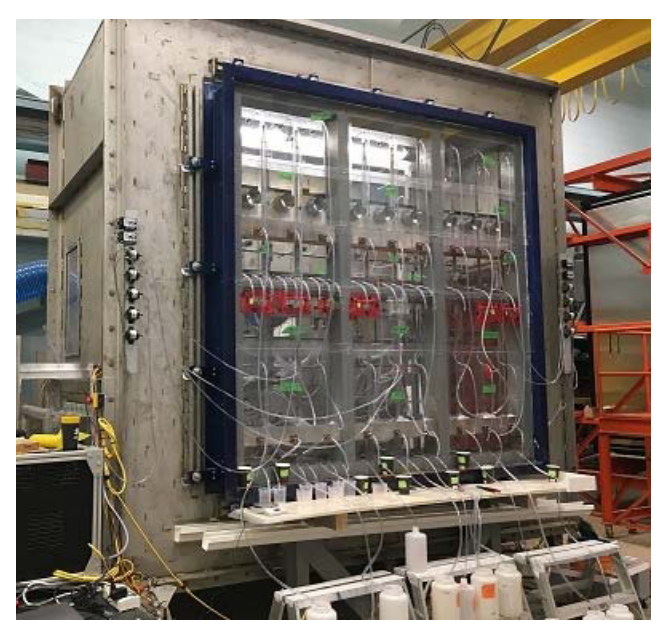

Fig. 1. Dynamic Wall Testing Facility.

\section{Analysis of wind-driven rain loads}

Hourly wind speed and hourly rainfall intensity of historical climate data collected from 1986 to 2016 from the Ottawa region were processed using the semiempirical WDR equation (Eq. 1) to generate WDR loads. Since other factors in the equation vary from case to case, they are considered as 1 for generalization. Hourly wind speed and hourly rainfall intensity yields the hourly WDR load. At the same time, the cumulative WDR load is also relevant as the moisture load obtained from an earlier time could be added to the subsequent moisture load.

Histograms in Fig.2 demonstrates the discrepancies of WDR load processed using different temporal schemes. The maximum WDR intensity from 1-hour WDR events is $34 \mathrm{~mm} / \mathrm{h}$ and the maximum WDR load obtained from such a short period event is $34 \mathrm{~mm}$. The maximum WDR intensity derived from 24-hour WDR events is $7.25 \mathrm{~mm} / \mathrm{h}$ and the maximum total WDR load for any consecutive 24hour event is $174 \mathrm{~mm}$. In terms of the watertightness test, applying an equivalent WDR load is more important than just simply using the WDR intensity.

Thus, the maximum annual WDR load over specific durations were calculated and used to generate the intensity-duration-frequency (IDF) plot to predict the extreme WDR loads for longer return periods. Probability distributions have been implemented to evaluate the risks of occurrence of extreme WDR load events and thereafter to determine the return period of these extreme events. The cumulative Generalized Extreme Value (GEV) distribution (3), which is usually used to model the maximum of random variables, and the cumulative Generalized Pareto (GPD) distribution (4), which is often used to describe scientific observable phenomena, were used for estimating the WDR load.

$$
F_{(\xi, \mu, \sigma)}(x)=1-\left(1+\frac{\xi(x-\mu)}{\sigma}\right)^{-\frac{1}{\xi}}
$$




$$
F_{(\xi, \mu, \sigma)}(x)=e^{-\left(1+\xi\left(\frac{x-\mu}{\sigma}\right)\right)^{-\frac{1}{\xi}}}
$$

Where $\xi$ is the shape factor, $\mu$ is the location factor and $\sigma$ is the scale factor.
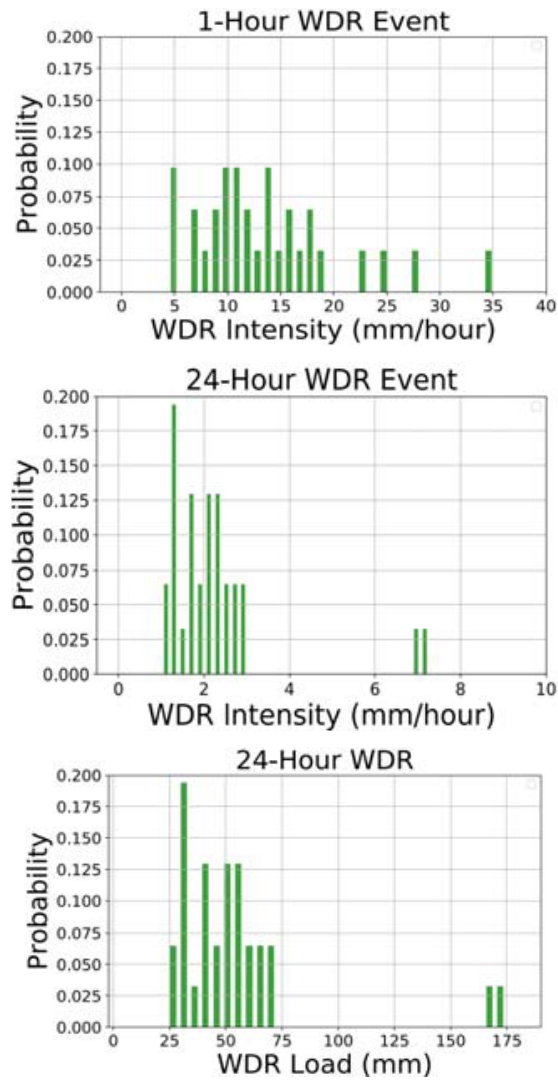

Fig. 2. WDR load histograms of different temporal schemes for the Ottawa Region, Canada.

\subsection{Co-occurrence of rain and wind}

According to several studies, including but not limited to [7-9], the degree of water infiltration is sensitive to both the water deposition rate, which represents the WDR load in the experiment, and the applied pressure, which is positively correlated with the wind speed. Therefore, an index which is the multiplication of the WDR load and concurrent wind pressure has been created to demonstrate the extent of water entry potential based on environmental conditions. The wind speed from the climate data was transferred into an equivalent wind dynamic pressure using the Bernoulli's equation (5) [R5]:

$$
P_{\text {dynamic }}=\frac{1}{2} \rho V_{e}^{2}
$$

Where $P_{\text {dynamic }}$ is the dynamic wind pressure, $\rho$ is the density of the flow and $V_{\mathrm{e}}$ is the flow velocity.

Table 1 depicts the top 13 most extreme hourly WDR events for 30 years of Ottawa climate data. As demonstrated, the most extreme WDR load event would not necessarily lead to the most extreme WDR-Pressure Index event as this also depends on the concurrent wind speed. Hence, an analysis similar to the IDF plot with respect to the WDR-Pressure Index was subsequently completed to estimate the return period of the extreme WDR-Pressure Index events. In addition, the WDRPressure Index can also be calculated and quantified during a watertightness test. For instance, the WDRPressure Index of a 10 minute watertightness test having a $3.4 \mathrm{~L} / \mathrm{min}-\mathrm{m}^{2}$ spray rate, and that deposits $34 \mathrm{~mm} / \mathrm{m}^{2}$ of water onto the test specimen over the test duration, in combination with a constant $100 \mathrm{~Pa}$ pressure applied to the specimen is equal to 3400 .

Table 1. WDR Load and WDR-Pressure Index for the Ottawa

\begin{tabular}{|c|c|c|c|c|c|}
\hline $\begin{array}{l}\text { Wind } \\
\text { Pressure } \\
\text { (Pa) }\end{array}$ & $\begin{array}{l}\text { Hourly } \\
\text { WDR Load } \\
\text { (mm) }\end{array}$ & $\begin{array}{c}\text { WDR x } \\
\text { Pressure } \\
\text { (Index) }\end{array}$ & $\begin{array}{l}\text { Wind } \\
\text { Pressure } \\
\text { (Pa) }\end{array}$ & $\begin{array}{l}\text { Hourly } \\
\text { WDR Load } \\
\text { (mm) }\end{array}$ & $\begin{array}{l}\text { WDR x } \\
\text { Pressure } \\
\text { (Index) }\end{array}$ \\
\hline 48 & 35 & 1678 & 141 & 27 & 3851 \\
\hline 141 & 27 & 3851 & 204 & 18 & 3743 \\
\hline 89 & 25 & 2198 & 121 & 22 & 2636 \\
\hline 35 & 23 & 793 & 121 & 20 & 2422 \\
\hline 33 & 22 & 730 & 89 & 25 & 2198 \\
\hline 121 & 22 & 2636 & 102 & 18 & 1817 \\
\hline 121 & 20 & 2422 & 48 & 35 & 1678 \\
\hline 45 & 19 & 847 & 111 & 12 & 1368 \\
\hline 204 & 18 & 3743 & 66 & 18 & 1190 \\
\hline 17 & 18 & 314 & 111 & 10 & 1139 \\
\hline 66 & 18 & 1190 & 111 & 10 & 1134 \\
\hline 46 & 18 & 830 & 81 & 13 & 1087 \\
\hline 102 & 18 & 1817 & 102 & 10 & 1057 \\
\hline
\end{tabular}
region, Canada.

\subsection{IDF plot and return period analysis}

An Intensity-Duration-Frequency plot (IDF) is a graphical representation that correlates rainfall intensity with its duration and frequency of occurrence. By using appropriate probability distribution functions, with data collected within a relatively short period of time, extreme events that have a longer return period can be estimated. Some modifications have been made to the IDF plot to comply with this study. The rainfall intensity in the conventional IDF plot was replaced by the WDR intensity, WDR load and the WDR-Pressure Index respectively. Discussion will be made for the fittings of GEV distribution functions and the Pareto distribution function as well as the new curves derived from the three new criteria.

The least square method was used for the fitting process for both probability distributions as were implemented. The annual maximum WDR load over 1hour, 2-hour, 4-hour, 8-hour, 12-hour and 24-hour periods were calculated and a frequency analysis was performed for each set of annual maxima to obtain the exceedance probability distribution functions.

In Table 2, parameters of each function for the two distributions are given for the respective durations considered. A larger value for the location factor $\mu$ and value for the scale factor $\sigma$ were both, respectively, for events having longer durations. WDR events with a long 
duration would tend to have a higher value for the lower limit of WDR load than WDR events of shorter duration. Consequently, the shape factor $\xi$ which describes the shape of tail of a distribution decreases when the duration increases. By using these parameters, the exceedance probability of more extreme WDR events can be estimated. Thereafter, the corresponding return period of these more extreme WDR events can be derived.

Table 2. Parameters of distributions for WDR intensity

\begin{tabular}{|c|c|c|c|}
\hline \multicolumn{4}{|c|}{ Generalized Extreme Value Distribution } \\
\hline Durations & $\mu$ & $\sigma$ & $\xi$ \\
\hline 1-Hour & 10.4 & 4.98 & 0.047 \\
\hline 2-Hour & 15.4 & 5.77 & 0.225 \\
\hline 4-Hour & 21.6 & 8.25 & -0.126 \\
\hline 8-Hour & 29.6 & 12.1 & -0.087 \\
\hline 12-Hour & 34.8 & 13.7 & -0.107 \\
\hline 24-Hour & 42.1 & 16.0 & -0.121 \\
\hline \multicolumn{4}{|c|}{ Generalized Pareto Distribution } \\
\hline Durations & $\mu$ & $\sigma$ & $\xi$ \\
\hline 1-Hour & 5.07 & 11.5 & -0.384 \\
\hline 2-Hour & 9.29 & 13.3 & -0.297 \\
\hline 4-Hour & 14.3 & 14.2 & -0.196 \\
\hline 8-Hour & 18.7 & 21.0 & -0.161 \\
\hline 12-Hour & 21.5 & 25.6 & -0.182 \\
\hline 24-Hour & 26.8 & 29.3 & -0.199 \\
\hline
\end{tabular}

The WDR Intensity-Duration-Frequency (WIDF) plot is depicted in Fig.6. WDR events with 2-year, 5-year, 10year, 20-year, 50-year and 100-year return periods were considered. Compared with a conventional IDF plot, this version uses the WDR intensity $(\mathrm{mm} / \mathrm{h})$ to replace the rainfall intensity of the IDF plot. The trend of the WIDF plot is similar to a conventional IDF plot in that WDR events having shorter durations will have higher intensity than WDR events of longer duration. Also, two sets of curves were generated using the parameters of the two distributions given in the WIDF plot. The WDR intensity derived from the GEV distribution is similar to, or lower than, the WDR intensity produced by the GPD distribution for the WDR events having more than a 5hour duration. For shorter duration WDR events, the GEV generated WDR intensity is higher than that generated by GPD distribution.

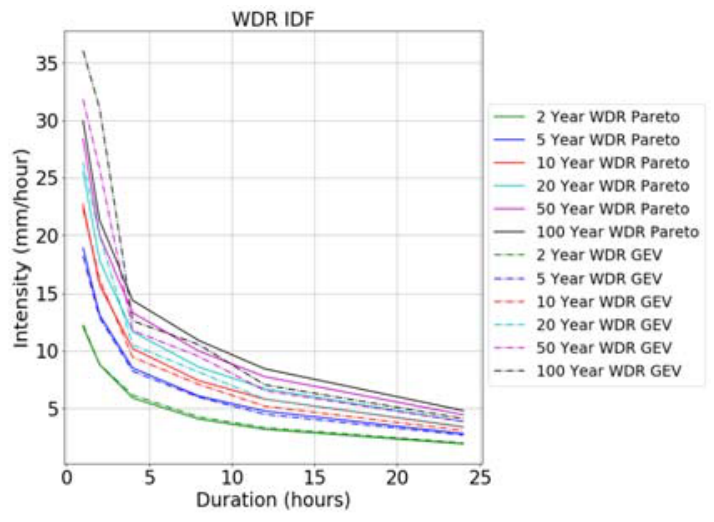

Fig. 6. WDR Intensity-Duration-Frequency (WIDF) plot for the Ottawa region, Canada.
In addition to the WIDF plot, the return period of WDR events with extreme WDR load $(\mathrm{mm})$ is also of interest. As shown in Fig.7, a WDR Load-DurationFrequency (WLDF) plot was developed by multiplying the WDR intensity with the corresponding event duration. With respect to the curve derived from the GPD distribution, WDR events of longer duration tend to have a higher WDR load which is reasonable and expected. The highest WDR load obtained from 24-hour WDR events with a 100 -year return period is $116 \mathrm{~mm}$. It is equivalent to undertaking a watertightness test over 35 minutes with a $3.4 \mathrm{~L} / \mathrm{min}-\mathrm{m}^{2}$ water deposition rate. From the shape of the curve, the WDR load tends to increase with a longer duration but with a lower increment.

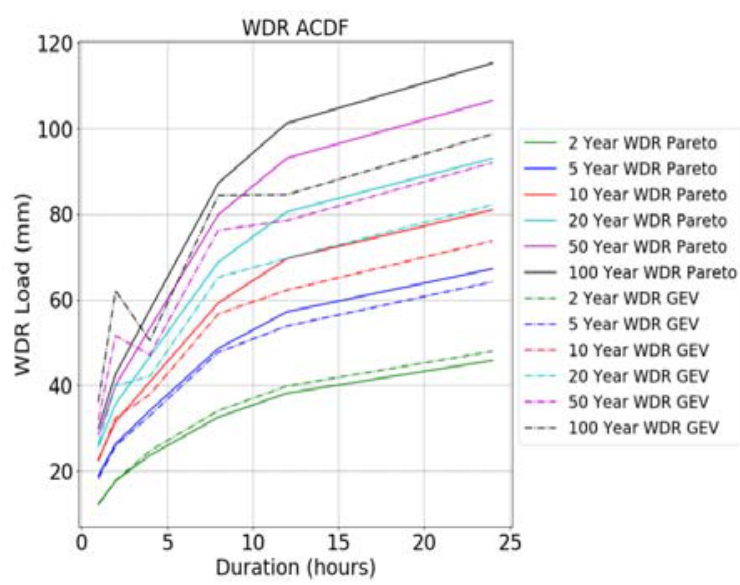

Fig. 7. WDR Load-Duration-Frequency (WLDF) plot for the Ottawa region, Canada.

The GEV distribution performs well to generate curves having a shorter return period. Spikes were observed in the curve derived from the GEV distributions for the WDR events with return periods greater than 20years. This indicates that the 2-hour WDR events collected from Ottawa climate data and from which a GEV distribution function was generated has a higher growth rate after the exceedance probability, which corresponds to a 20-year return period, than that generated by WDR events having other durations. Climate data collected for other regions will be used to validate this abnormality. For the rest of the GEV distribution curves that were generated, the WDR load is lower than the curves produced using the GPD distribution with the exception for that curve having a 2-year return period which provided a higher WDR load.

Table 3. Parameters of distributions for WDR-Pressure Index

\begin{tabular}{|c|c|c|c|}
\hline \multicolumn{4}{|c|}{ Generalized Extreme Value Distribution } \\
\hline Durations & $\mu$ & $\sigma$ & $\xi$ \\
\hline 1-Hour & 374 & 217 & 0.646 \\
\hline 2-Hour & 561 & 276 & 0.751 \\
\hline 4-Hour & 760 & 406 & 0.812 \\
\hline 8-Hour & 1020 & 585 & 0.651 \\
\hline 12-Hour & 1170 & 673 & 0.425 \\
\hline 24-Hour & 1330 & 760 & 0.292 \\
\hline
\end{tabular}




\begin{tabular}{|c|c|c|c|}
\hline \multicolumn{4}{|c|}{ Generalized Pareto Distribution } \\
\hline Durations & $\mu$ & $\sigma$ & $\xi$ \\
\hline 1-Hour & 158 & 426 & 0.207 \\
\hline 2-Hour & 250 & 634 & 0.077 \\
\hline 4-Hour & 358 & 855 & 0.133 \\
\hline 8-Hour & 438 & 1240 & 0.006 \\
\hline 12-Hour & 505 & 1430 & -0.106 \\
\hline 24-Hour & 560 & 1890 & -0.112 \\
\hline
\end{tabular}

The same type of frequency analysis was also carried out for the newly derived WDR-Pressure-Index. For both distributions, the value of the location factor $\mu$ and scale factor $\sigma$ were found to increase with an increase in duration whilst the value of the shape factor $\xi$ decreased. These parameters were also used to estimate the probability of occurrence of events for specific values of WDR-Pressure Index of interest to this study.

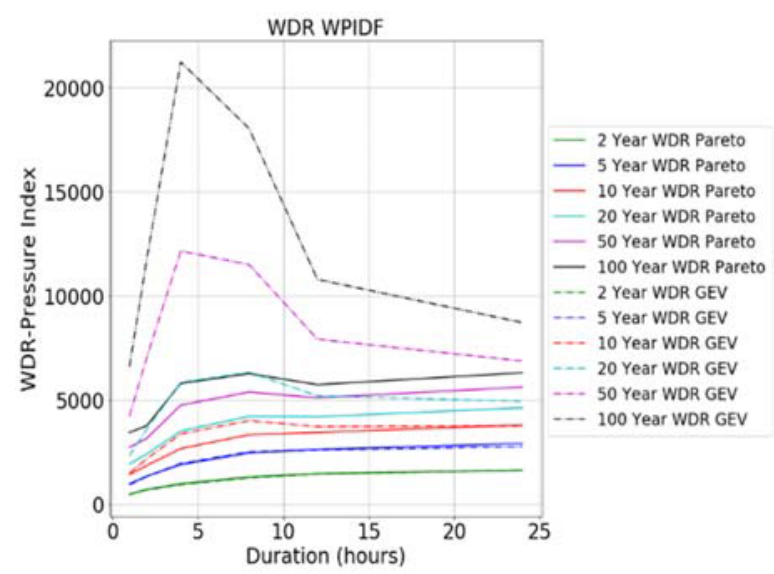

Fig. 8. WDR-Pressure Index-Duration-Frequency (WPIDF) plot for the Ottawa region, Canada.

The probability of occurrence obtained above was used to derive the corresponding return period to generate a WDR-Pressure Index-Duration-Frequency (WPIDF) plot. Compared with the WLDF plot, the GPD distribution generated flatter curves for all return periods and small bulges were observed on those curves. This type of 'bulge' was not found on curves of the WLDF plot. As such, and given that the wind pressure is the additional variation to the WLDF curves, it is supposed that this is the cause of changes to the WLDF plot. Moreover, the occurrence of 'bulges' evident of all the curves exists between 2-hour and 12-hour durations and the reason for this is that in the Ottawa region, extreme wind events usually only last a few hours. So for WDR events of longer duration, the contribution of the wind to the WDRPressure Index would be smaller than that for WDR events of shorter duration. The GEV distribution generated curves have the same issue as that for the WLDF plot. It will be further validated with the climate data from other regions in Canada.

\section{Conclusions}

New plots of WDR Intensity-Duration-Frequency (WIDF) using both a GEV and GPD distributions as derived from modifying IDF plots using WDR intensity look normal. The GEV distribution generates a slightly higher WDR intensity than those obtained from the GPD distribution. However, for the WLDF and WPIDF plots, at shorter durations the GEV distribution displays large spikes. Additional observational data will be used to test the GEV distribution in a subsequent project. However, the GPD distribution will be chosen to undertake a frequency analysis and to estimate WDR events having a longer return period. Goodness-of-fit tests of the distribution will also be added to this analysis.

The severity of a WDR event still could not be fully determined using the WDR-pressure Index approach, as the susceptibility of the rate of water entry of the wall assembly to the water deposition rate and applied pressure may vary for different configurations of wall assemblies and different types of deficiencies. Empirical coefficients will be developed to improve the applicability and accuracy of the WDR-pressure index.

An investigation of the WDR load and the WDRPressure Index at longer durations will be carried out as curves in the WLDF plot and WPIDF plots tend to become flat for long duration events. Ideally, the extreme WDR load will be selected from the plot where the curve does not vary much with duration while the length of duration can still be considered as a consecutive WDR event.

\section{References}

1. Lacy R. E., Build. Res. Establish. Report. (1977)

2. Straube J., Burnett E., Proc. Int. Build. Phys. Confer. 375-382 (2000)

3. ISO Standard, 15927-3 (2009)

4. B. Blocken, J. Carmeliet, J. Wind Eng. Indus. Aero. 93, 1079-1130 (2004)

5. H. Ge, U. K. D. Nath, V. Chiu, Build. And Enviro. 116, 229-245 (2017)

6. M. Abuku, B. Blocken, J. Poesen, S. Roels, $11^{\text {th }}$ Amer. Conf. Wind Eng.

7. M. A. Lacasse, H. Miyauchi, J. Hiemstra, J. ASTM Int. 6, 6 (2009)

8. N. Sahal, M. A. Lacasse, National Research Council Canada Public. Arch. (2005)

9. N. V. D. Bossche, S. V. Goethem, S. Scharlaken, S. Sulmon, A. Janssens, ISBP, 24-27, 431-438 (2015) 